\title{
INEQUALITIES AND A LIMIT THEOREM FOR CERTAIN WIENER INTEGRALS
}

J. YEH ${ }^{1}$

The Wiener space $C_{w}$ of real-valued continuous functions $x(t)$ on $[0,1]$ with $x(0)=0$ is an inner product space with respect to the inner product

$$
\langle x \mid y\rangle=\int_{0}^{1} x(t) y(t) d t, \quad x, y \in C_{w} .
$$

Let $\|x\|, x \in C_{w}$, be the associated Hilbert norm. According to Cameron and Martin [1],

$$
\int_{C_{w}} \exp \left\{\lambda\|x\|^{2}\right\} d_{w} x=\left(\sec \lambda^{1 / 2}\right)^{1 / 2}, \quad 0 \leqq \lambda<\frac{\pi^{2}}{4} .
$$

In the present paper we consider

$$
\int_{C_{w}} \exp \{\lambda\langle x \mid y\rangle\} d_{w} x
$$

Our result is the following

THEOREM 1. Let $f(u)$ be a real-valued Lebesgue measurable function such that $\int_{-\infty}^{\infty} f(u) e^{-u^{2}} d u$ converges absolutely as a Lebesgue integral. Then for any $y \in L_{2}[0,1]$ and complex number $\mu$

$$
\begin{aligned}
\int_{C_{\omega}} f & {[x(1)] \exp \{\mu\langle x \mid y\rangle\} d_{w} x } \\
= & \frac{1}{\pi^{1 / 2}} \exp \left\{\frac{\mu^{2}}{4}\left(\|Y\|^{2}-[Z(1)]^{2}\right)\right\} \\
& \cdot \int_{-\infty}^{\infty} f(u) \exp \left\{-u^{2}+\mu[Y(1)-Z(1)] u\right\} d u
\end{aligned}
$$

where

(2) $\quad Y(t)=\int_{0}^{t} y(s) d s, \quad Z(t)=\int_{0}^{t} Y(s) d s, \quad t \in[0,1]$.

Received by the editors March 23, 1964.

1 This research was supported in part by the National Science Foundation Grant GP 1620. 
2. In particular

(3) $\int_{C_{w}} \exp \{\mu\langle x \mid y\rangle\} d_{w} x=\exp \left\{\frac{\mu^{2}}{4}\left(\|Y\|^{2}-2 Z(1) Y(1)+[Y(1)]^{2}\right)\right\}$.

3. Furthermore for any real $\alpha$,

$$
\exp \left\{\frac{\alpha^{2}}{4}\left\langle x_{0} \mid y\right\rangle^{2}\right\} \leqq \int_{C_{w}} \exp \{\alpha\langle x \mid y\rangle\} d_{w} x \leqq \exp \left\{\alpha^{2}\|y\|^{2}\right\},
$$

(5) $\exp \left\{-\alpha^{2}\|y\|^{2}\right\} \leqq \int_{C_{w}} \exp \{i \alpha\langle x \mid y\rangle\} d_{w} x \leqq \exp \left\{-\frac{\alpha^{2}}{4}\left\langle x_{0} \mid y\right\rangle^{2}\right\}$

where $x_{0}(t)=t$ on $[0,1]$.

Corollary 1. For $0 \leqq \alpha<\pi / 2$

$$
\left|\int_{C_{w} \times C_{w}} \exp \{\alpha\langle x \mid y\rangle\}\left(d_{w} x\right) \times\left(d_{w} y\right)\right| \leqq(\sec \alpha)^{1 / 2}
$$

where the integral is a double Wiener integral on $C_{w} \times C_{w}$ with respect to the product measure.

CoROllaRy 2. Let $\alpha$ be real. Then for any $\left\{y_{n}\right\} \subset C_{w}$ such that $\lim _{n \rightarrow \infty}\left|\left\langle x_{0} \mid y_{n}\right\rangle\right|=\infty$,

$$
\lim _{n \rightarrow \infty} \int_{C_{w}} \exp \left\{i \alpha\left\langle x \mid y_{n}\right\rangle\right\} d_{w} x=0 .
$$

Proof of The Theorem. For $y \in C_{w}, Y(t)$ as defined by (2) is of bounded variation and furthermore $Y^{\prime}(t)=y(t)$ almost everywhere on $[0,1]$. Thus for any $x \in C_{w}$, by integration by parts

(8) $\int_{0}^{1} x(t) y(t) d t=\int_{0}^{1} x(t) d Y(t)=Y(1) \int_{0}^{1} d x(t)-\int_{0}^{1} Y(t) d x(t)$.

If $y(t) \equiv 0$ almost everywhere on $[0,1],\langle x \mid y\rangle=0$ for all $x \in C_{w}$ and (1) holds trivially. Assume that $y(t)$ is not almost identically vanishing on $[0,1]$. Then $Y(t)$ is not constant on $[0,1]$ so that the function which is identically equal to 1 on $[0,1]$ and $Y$ are linearly independent on $[0,1]$. By the Gram-Schmidt procedure we obtain an equivalent orthonormal system consisting of the function identically equal to 1 and

$$
W(t)=\frac{1}{\left(\|Y\|^{2}-[Z(1)]^{2}\right)^{1 / 2}}\{Y(t)-Z(1)\}
$$


where $Z(t)$ is as defined by (2). Now solving (9) for $Y(t)$ and substituting the result in (8) we have

$$
\int_{0}^{1} x(t) y(t) d t=c_{1}(y) \int_{0}^{1} d x(t)+c_{2}(y) \int_{0}^{1} W(t) d x(t)
$$

where

$$
c_{1}(y)=Y(1)-Z(1), \quad c_{2}(y)=-\left(\|Y\|^{2}-[Z(1)]^{2}\right)^{1 / 2}
$$

According to Paley-Wiener [2], if $\left\{\alpha_{k}(t)\right\}$ is an orthonormal set of real valued functions of bounded variation on $[0,1]$,

$$
\begin{aligned}
\int_{C_{w}} \phi\left[\int_{0}^{1} \alpha_{1}(t) d x(t), \cdots,\right. & \left.\int_{0}^{1} \alpha_{n}(t) d x(t)\right] d{ }_{w} x \\
= & \frac{1}{\pi^{n / 2}} \int_{-\infty}^{\infty} \cdots \int_{-\infty}^{\infty} \phi\left(u_{1}, \cdots, u_{n}\right) \\
& \cdot \exp \left\{-\left(u_{1}^{2}+\cdots+u_{n}^{2}\right)\right\} d u_{1} \cdots d u_{n}
\end{aligned}
$$

for every Lebesgue measurable function $\phi\left(u_{1}, \cdots, u_{n}\right)$ for which the integral on the right side converges absolutely as a Lebesgue integral. Applying this result together with (10) and

$$
\int_{-\infty}^{\infty} \exp \left\{-s^{2}+a s\right\} d s=\pi^{1 / 2} \exp \left\{\frac{a^{2}}{4}\right\},
$$

we have

$$
\begin{aligned}
& \int_{C_{w}} f[x(1)] \exp \{\mu\langle x \mid y\rangle\} d_{w} x \\
& =\frac{1}{\pi} \int_{-\infty}^{\infty} f(u) \exp \left\{\mu c_{1}(y) u-u^{2}\right\} d u \cdot \int_{-\infty}^{\infty} \exp \left\{\mu c_{2}(y) v-v^{2}\right\} d v \\
& =\frac{1}{\pi^{1 / 2}} \exp \left\{\frac{\mu^{2}}{4}\left[c_{2}(y)\right]^{2}\right\} \int_{-\infty}^{\infty} f(u) \exp \left\{\mu c_{1}(y) u-u^{2}\right\} d u,
\end{aligned}
$$

which is (1).

Choosing $f(u) \equiv 1$, we have

$$
\begin{aligned}
\int_{C_{w}} \exp \{\mu\langle x \mid y\rangle\} d_{w} x & =\exp \left\{\frac{\mu^{2}}{4}\left[c_{2}(y)\right]^{2}\right\} \exp \left\{\frac{\mu^{2}}{4}\left[c_{1}(y)\right]^{2}\right\} \\
& =\exp \left\{\frac{\mu^{2}}{4}\left(\|Y\|^{2}-2 Z(1) Y(1)+[Y(1)]^{2}\right)\right\}
\end{aligned}
$$


according to (11). This proves (3).

To prove (4), (5) we remark that according to Schwarz's inequality, $|Z(1)| \leqq\|Y\|$ so that

$$
\begin{aligned}
\{Z(1)-Y(1)\}^{2} & \leqq\|Y\|^{2}-2 Z(1) Y(1)+[Y(1)]^{2} \\
& \leqq\{\|Y\|+|Y(1)|\}^{2} .
\end{aligned}
$$

Again by Schwarz's inequality we have $|Y(1)| \leqq\|y\|$ and also

$$
\|Y\|^{2}=\int_{0}^{1}|Y(t)|^{2} d t=\int_{0}^{1}\left|\int_{0}^{t} y(s) d s\right|^{2} d t \leqq\|y\|^{2}
$$

so that

$$
\{\|Y\|+|Y(1)|\}^{2} \leqq 4\|y\|^{2} .
$$

On the other hand

$$
Z(1)=\int_{0}^{1} Y(t) d t=\int_{0}^{1}\left\{\int_{0}^{t} y(s) d s\right\} d t=\int_{0}^{1} y(s)(1-s) d s
$$

so that

$$
Z(1)-Y(1)=-\int_{0}^{1} s y(s) d s=-\left\langle x_{0} \mid y\right\rangle
$$

where $x_{0}(t)=t$ on $[0,1]$. Thus combining (12), (13), (14) we have

$$
\left\langle x_{0} \mid y\right\rangle^{2} \leqq\|Y\|^{2}-2 Z(1) Y(1)+[Y(1)]^{2} \leqq 4\|y\|^{2} .
$$

Now (4), (5) follow from (3), (15). This completes the proof of the theorem.

Corollary 1 follows from (4) by means of Fubini's Theorem and (0). Corollary 2 follows from (5).

\section{BIBLIOGRAPHY}

1. R. H. Cameron and W. T. Martin, Transformations of Wiener integrals under a general class of linear transformations, Trans. Amer. Math. Soc. 58 (1945), 184-219.

2. R. E. A. C. Paley and N. Wiener, Fourier transforms in the complex domain, Amer. Math. Soc. Colloq. Publ. Vol. 19, Amer. Math. Soc., Providence, R.I., 1934.

NEW YoRK UNIVERSITY AND

UNIVERSITY OF ROCHESTER 